\title{
Segmentation of Salient Objects in Digital Image using Hybridization Gradient Based Technique
}

\author{
Sandeep ${ }^{1}$, Suresha $\mathrm{M}^{2}$ \\ Department of Computer Science ${ }^{1,2}$, Kuvempu University ${ }^{1,2}$ \\ Email: sandeepgowda33@gmail.com,srit_suresh@yahoo.com²
}

\begin{abstract}
Segmentation of salient objects is essential application for classification of image, recognition of object and CBIR tasks. In this paper, authors implement a new approach for segmentation of salient region from a digital image using hybridization gradient techniques. Here authors project, smoothing a low intensity values using L0 gradient minimization technique. After smoothing an image, applying gradient based segmentation. In experimental results, we use Flowers-17 database to check the effectiveness of the proposed approach. Authors show that proposed approach outperforms with gradient methods using recall, precision and F1-score.
\end{abstract}

Index Terms- Flower-17 Datasets, F1- Score, Gradient Based Method, $\mathrm{L}_{0}$ Gradient Minimization, Precision, Recall.

\section{INTRODUCTION}

Image segmentation is an important procedure in image analysis, and it splits an image into its essential parts or objects in the image. The level of segmentation build on the problem of application. Segmentation method for images usually solves using two categories such as discontinuity and similarity of image intensity values. A rapid change in intensity comes in similarity and discontinuity category based on image partition into region [7][3]. Edge detection is a part of segmentation and it comes under discontinuity category. They are deals with changes in the pixel which differentiate object boundaries in an image [4]. Smoothing techniques useful in many applications such as image recognition, object classification and segmentation, numerous photo editing and non-photorealistic rendering tasks whereas smoothing techniques minimizing low amplitude values [8]. Always edge detection finds some sharp edges. when image is fully smoothing Gradient method is suitable for segmentation. It clearly identifies magnitude edges in $\mathrm{x}$ and $\mathrm{y}$-direction gradient intensity distribution, it computes the overlap of image gradient with the boundaries determined by intensity distribution through introducing a probability offset to intensity distribution. The maximum overlap indicates the optimal boundaries of the interested objects [2].

In the overview of this paper, Authors describe review of segmentation using gradient method in Section 2. The segmentation using Hybridization Gradient Techniques is designed in Section 3, Then, presented proposed experimental results discussed in Section 4. Lastly, in section 5 conclusion of our work.

\section{RELATED WORK}

In [7], proposed a modern technique related on segmentation in image processing, Analysis and Machine Vision techniques. In [Madhuri], presented a deep introduction to the fundamental concepts and methodologies of image processing. In [5], proposed classification of flower-17 dataset using bag of visual words model. In this paper, created the visual vocabulary in various aspects like color, shape, and texture. Experiment was conducted flower-17 dataset and it contains 1360 images in 17 categories of flower. In [8], proposed a new image editing method for natural images. The main aim of this work is sharpening major edges and eliminating minor edge of images using $\mathrm{L}_{0}$ gradient minimization techniques. In [4], presented an edge detection technique for segmentation and comparison is done in experiment using matlab software. In [2], proposed a level set method using gradient information of image. It can tolerate estimation error in intensity distribution and detect object boundaries whose gradient is low and experimental results proved that proposed approach produces efficient results of segmentation on public lung image data. In [6], presented an image analysis based on first-order or second-order derivatives techniques. In this work, gradient method using edge detection is used on natural images. In [1], proposed novel techniques for preserving edges using multi- 


\section{E-ISSN: 2321-9637}

\section{Available online at www.ijrat.org}

scale image decompositions. This works based on bilateral filter for edge-preserving .

\section{PROPOSED METHODOLOGY}

To the best of authors knowledge, it is a novel work for segmentation of digital images using Hybridization Gradient based Techniques. The proposed methodology is shown in the Fig. 1. In this approach, smoothing an original image using L0 gradient minimization techniques. After smoothing an image Sobel operator used for gradient based segmentation. Kernel Sobel shown in Fig. 3 .. $\mathrm{L}_{0}$ gradient minimization technique is operative on major edges sharpening by growing the gradient of transition whereas eliminating a low-amplitude structure. It can control globally and count non-zero gradients result into the eminent structure in a sparsity approach. Edge-preserving smoothing approaches shown in Fig. 2 [8].

\section{1. $L_{0}$ Gradient Minimization Technique}

Image representation of $2 \mathrm{D}$, I symbolize the input image and $\mathrm{S}$ denotes the resultant image. The gradient $\nabla S_{p}=\left(\partial_{x} S_{p}, \partial_{y} S_{p}\right)^{T}$ is gradient for each pixel $\mathrm{p}$ and calculated as color change between next to pixels of $x$ and y directions [8] [1]. shown in equation-(1).

$$
C(S)=\#\left\{p|| \partial_{x} S_{p}|+| \partial_{y} S_{p} \mid \neq 0\right\}
$$

It calculate number of $p$ and its magnitude $\left|\partial_{x} S_{p}\right|+$ $\left|\partial_{y} S_{p}\right|$ is not zero and $S$ is estimation.

$$
\min _{s}\left\{\sum_{p}\left(S_{p}-I_{p}\right)^{2}+\lambda . C(S)\right\}
$$

In the concept of color images, $\left|\partial S_{p}\right|$ denote summation of gradient magnitudes in color image. The expression $\Sigma(\mathrm{S}-\mathrm{I}) 2$ gives structure similarity of an image [Farbman].

Equation-(2) produced discrete counting metric. So that, it is very problematic to solve by the two reason respectively i.e., global discontinuity statistically and pixel-wise difference. To solve this problem, implemented an optimization strategy with halfquadratic splitting techniques Based on auxiliary variables $h_{p}$ and $v_{p}$ develop original images update iteratively.

$$
\begin{aligned}
& \min _{s, h, v}\left\{\sum_{p}\left(S_{p}-I_{p}\right)^{2}+\lambda \cdot C(h, v)\right. \\
& +\beta\left(\left(\partial_{x} S_{p}-h_{p}\right)^{2}\right. \\
& \left.\left.+\left(\partial_{y} S_{p}-v_{p}\right)^{2}\right)\right\}
\end{aligned}
$$

Where $C(h, v)=\#\left\{p|| h_{p}|+| v_{p} \mid \neq 0\right\}$ and $\beta$ an parameter to control the similarity between auxiliary variables $(h, v)$ and their similar gradients. Equation-(3) is shows alternatively minimizing $(h, v)$ and $S$.

Subproblem 1: To computing $S$ : The equation is quadratic and therefore has a global minimum even by gradient decent. Instead, transform derivative operators afterward Fast Fourier Transform (FFT) for speedup. This yields solution

$$
\begin{gathered}
\min _{p}\left\{\sum_{p}\left(S_{p}-I_{p}\right)^{2}+\beta\left(\left(\partial_{x} S_{p}-h_{p}\right)^{2}+\left(\partial_{y} S_{p}-v_{p}\right)^{2}\right)\right\}, \\
S=\mathcal{F}^{-1} \frac{\mathcal{F}(I)+\beta\left(\mathcal{F}\left(\partial_{x}\right)^{*} \mathcal{F}(h)+\mathcal{F}\left(\partial_{y}\right)^{*} \mathcal{F}(v)\right)}{\mathcal{F}(1)+\beta\left(\mathcal{F}\left(\partial_{x}\right)^{*} \mathcal{F}\left(\partial_{x}\right)+\mathcal{F}\left(\partial_{y}\right)^{*} \mathcal{F}\left(v \partial_{y}\right)\right)}
\end{gathered}
$$

Subproblem 2: computing $(\mathrm{h}, \mathrm{v})$ The objective function for $(\mathrm{h}, \mathrm{v})$ is

$\min _{h, v}\left\{\sum_{p}\left(\left(\partial_{x} S_{p}-h_{p}\right)^{2}+\left(\partial_{y} S_{p}-v_{p}\right)^{2}\right)+\frac{\lambda}{\beta} C(h, v)\right\}$,

$$
\begin{gathered}
\sum_{p}^{\min } h_{p} v_{p}\left\{\sum_{p}\left(\left(h_{p}-\partial_{x} S_{p}\right)^{2}+\left(v_{p}-\partial_{y} S_{p}\right)^{2}\right)+\frac{\lambda}{\beta} H\left(\left|h_{p}\right|\right.\right. \\
\left.\left.+\left|v_{p}\right|\right)\right\}
\end{gathered}
$$

$E_{p}=\left\{\left(\left(h_{p}-\partial_{x} S_{p}\right)^{2}+\left(v_{p}-\partial_{y} S_{p}\right)^{2}\right)+\frac{\lambda}{\beta} H\left(\left|h_{p}\right|+\right.\right.$ $\left.\left.\left|v_{p}\right|\right)\right\}$,

$$
\left(h_{p}, v_{p}\right)=\left\{\begin{array}{c}
(0,0) \quad\left(\partial_{x} S_{p}\right)^{2}+\left(\partial_{y} S_{p}\right)^{2} \leq \frac{\lambda}{\beta} \\
\left(\partial_{x} S_{p}, \partial_{y} S_{p}\right) \quad \text { otherwise }
\end{array}\right.
$$

(2) Proof,

When $\frac{\lambda}{\beta} \geq\left(\partial_{x} S_{p}\right)^{2}+\left(\partial_{y} S_{p}\right)^{2}$, non-zero $\left(h_{p}, v_{p}\right)$ yields

$$
\begin{array}{cl}
E_{p}=\left(\left(h_{p}, v_{p}\right) \neq(0,0)\right)= & \left(h_{p}-\partial_{x} S_{p}\right)^{2}+\left(v_{p}-\partial_{y} S_{p}\right)^{2}+\frac{\lambda}{\beta} \\
& \geq \frac{\lambda}{\beta} \\
& \\
& \geq\left(\partial_{x} S_{p}\right)^{2}+\left(\partial_{y} S_{p}\right)^{2}
\end{array}
$$

Note that $\left(h_{p}, v_{p}\right)=(0,0)$ lead to

$$
E_{p}\left(\left(h_{p}, v_{p}\right)=(0,0)=\left(\partial_{x} S_{p}\right)^{2}+\left(\partial_{y} S_{p}\right)^{2}\right)
$$

Comparing Equation (10) and (11), the minimum energy $E_{p}^{*}=\left(\partial_{x} S_{p}\right)^{2}+\left(\partial_{y} S_{p}\right)^{2}$ is produced when $\left(h_{p}, v_{p}\right)=$ $(0,0)$. 


\section{Available online at www.ijrat.org}

Algorithm for L0 Gradient Minimization

Input: image $\mathrm{I}$, smoothing weight $\lambda$, parameters

$\beta 0, \beta \max , \quad$ and rate $\kappa$

Initialization: $S \leftarrow I, \beta \leftarrow \beta 0, i \leftarrow 0$

repeat

With S(i), solve for h(i)

$\mathrm{p}$ and $\mathrm{v}(\mathrm{i})$

p in Eq. (9).

With h(i) and v(i), solve for $\mathrm{S}(\mathrm{i}+1)$ with Eq. (5).

$\beta \leftarrow \kappa \beta, \mathrm{i}++$.

until $\beta \geq \beta \max$

Output: result image $S$.

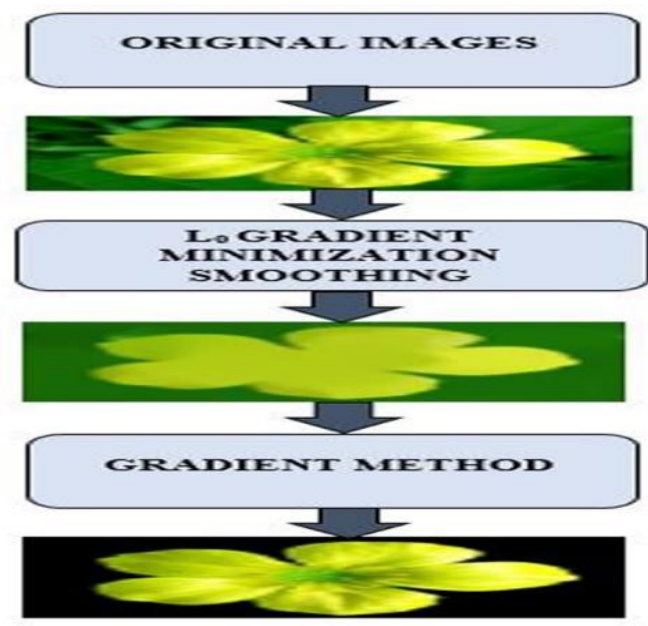

Fig.1: Proposed methodology for

Segmentation of salient objects.

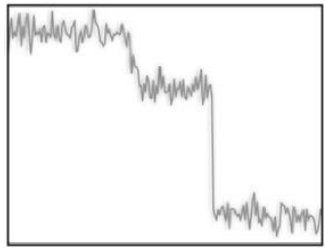

(a)

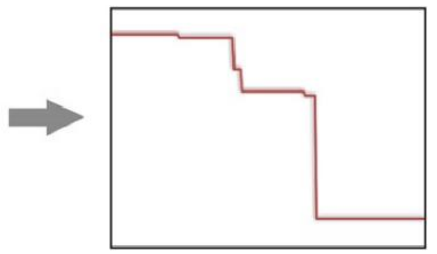

(b)
Fig.2: Smoothing edges using L0 gradient (a) Significant edges (b)Suppression of significant edges.

\subsection{Gradient Based Technique}

The Sobel edge detection significantly detect local variation in image intensity level. The intensity variation is measured by the image gradient. Since an image $f(x, y)$ is a two-dimensional and its gradient is form of vector. The vector and gradient magnitude computed in $\mathrm{x}$ and $\mathrm{y}$-direction shown in Equation (10)(11).

$$
f=\sum_{i=1}^{i} \sum_{j=1}^{j} f(x, y)
$$

$$
\begin{array}{r}
\overrightarrow{\nabla f}=\left[\begin{array}{l}
G_{x} \\
G_{y}
\end{array}\right]=\left[\begin{array}{c}
\frac{\partial f}{\partial x} \\
\frac{\partial f}{\partial y}
\end{array}\right], \\
\text { where } \begin{aligned}
\overrightarrow{\nabla f} & =\text { Gradient vector } \\
G_{x} & =\text { Gradient of x-direction } \\
G_{y} & =\text { Gradient of y-direction } \\
\nabla f & =\operatorname{mag}(\overrightarrow{\nabla f}) \\
|G| & =\sqrt{G_{x}^{2}+G_{y}^{2}}
\end{aligned}
\end{array}
$$

$|G|=\left|G_{x}\right|+\left|G_{y}\right|$

Where $\nabla f=$ Magnitude of gradient vector

$\left|G_{x}\right|=$ Magnitude of gradient $\mathrm{x}$-direction

$\left|G_{y}\right|=$ Magnitude of gradient $\mathrm{y}$-direction

$$
\operatorname{Grad}=\max (\nabla f)
$$

where $\mathrm{Grad}=$ maximum of magnitude of gradient.

$$
h(\mathrm{Grad})=\operatorname{hist}(\mathrm{Grad})
$$

Where $h(\mathrm{Grad})=$ histogram of Magnitude of gradient vector

Where

$$
Q=\operatorname{prctile}(h(\mathrm{Grad}), 0.1)
$$

$Q=$ Percentiles of a data set histogram of Magnitude of gradient vector

$$
\begin{gathered}
\text { marker image }=\text { Grad }>Q \\
h(\text { marker image })=\text { hist }(\text { marker image })
\end{gathered}
$$

where $h($ marker image $)=$ histogram of marker image

$$
\text { Tresh }=\left\{\begin{array}{c}
1 \text { if } h(\text { marker image }) \geq T \\
0 \text { otherwise }
\end{array}\right.
$$

where Tresh is a thresholded image $h$ (marker image) at global threshold $T$. Next step image converted into black and white image using Tresh value finally convolution function applied for original and Otsuthresh (Tresh) image after obtaining segmented image.

\section{RESULT AND DISCUSSION}

In this work, proposed approach on flower-17 category benchmark dataset is used [5] and considered 321 images for experiment. Fig 4 displays the Comparison of Gradient method and proposed method. Fig. 5 shows experimental result in graphical representation.

\subsection{Precision and Recall and F1-Score}

In experimental results, Hybridization Gradient Technique and traditional Gradient Techniques is comparison of accuracy using Precision and Recall and F1-Score. In this work, the target region segmentation is called True Positive (TP) class, non-target region segmentation is called True Negative (TN) class. 


\section{Available online at $w w w . i j r a t . o r g$}

$$
\begin{gathered}
\text { Precision }=\frac{T P}{T P+F P} \\
\text { Recall }=\frac{T P}{T P+F N} \\
F 1 \text { Score }=2 * \frac{(\text { Precision } * \text { Recall })}{(\text { Precision }+ \text { Recall })}
\end{gathered}
$$

Table 1. Evaluate performance of proposed method with Gradient technique.

\begin{tabular}{|l|l|l|l|l|}
\hline Sl.no & Techniques & Precision & Recall & $\begin{array}{l}\text { F1 } \\
\text { Score }\end{array}$ \\
\hline 1 & Proposed method & 57.43 & 98.25 & 80.02 \\
\hline 2 & $\begin{array}{l}\text { Gradient based } \\
\text { method }\end{array}$ & 52.10 & 94.08 & 75.57 \\
\hline
\end{tabular}

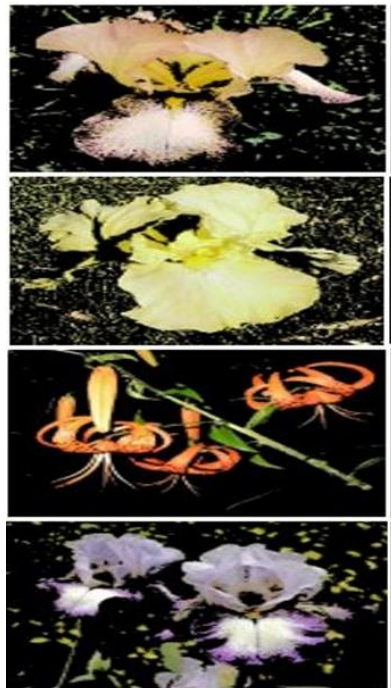

(a)

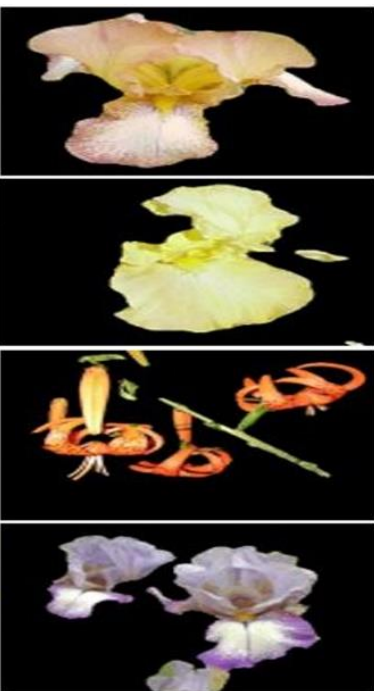

(b)
Fig. 4: Comparison of Gradient based segmentation and proposed method (a)Gradient method (b) proposed method.

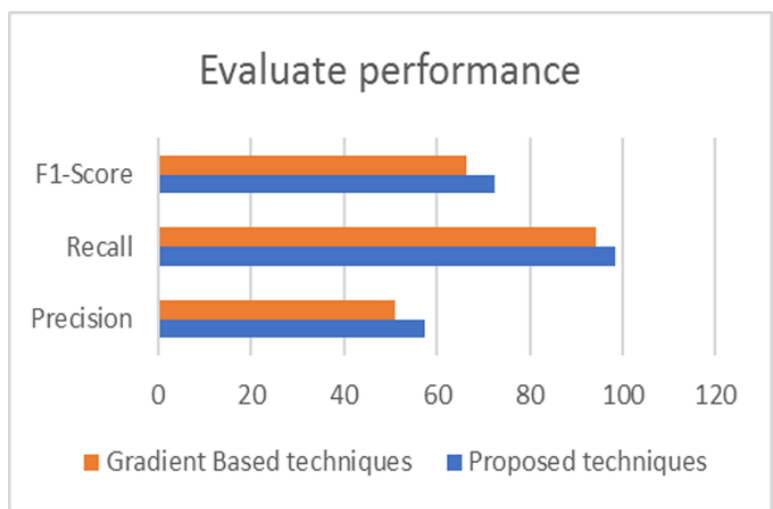

Fig. 5: Comparison of proposed techniques with Gradient based technique.

\section{CONCLUSION}

In this paper, Authors proposed a novel approach hybridization of gradient based technique for segmentation of salient objects. First, existing editing tool $\mathrm{L}_{0}$ gradient minimization techniques significantly supportive for describing and enhancing essential image elements, i.e., salient sharpening major edges and diminishing insignificant minor edge details. It relays in essence to edge-preserving smoothing. Second, gradient method applies for $\mathrm{L}_{0}$ gradient minimization smoothing images. finally, obtained segmented images and it depend on important global edges. Experimentally demonstrates that proposed method for Flowers-17 dataset images, it shows good segmentation results compares to the traditional gradient based method.

\section{REFERENCES}

[1] Farbman, Z; Fattal, R; Lischinski, D; Szeliski, R. (2008): Edge-preserving decompositions for multi-scale tone and detail manipulation. In ACM Transactions on Graphics, 27(3), pp. 67.

[2] Liu, S; Li, J. (2006): Automatic medical image segmentation using gradient and intensity combined level set method. In Engineering in Medicine and Biology Society, 2006. EMBS'06. 28th Annual International Conference of the IEEE, pp. 3118-3121.

[3] Madhuri, J. A ; (2006): Digital Image Processing, Prentice Hall.

[4] Muthukrishnan, R; Radha, M. (2011): Edge detection techniques for image segmentation. International Journal of Computer Science \& Information Technology, 3(6), pp.259.

[5] Nilsback, M. E ; Zisserman, A. (2006): A visual vocabulary for flower classification, In Computer Vision and Pattern Recognitio, Computer Society Conference on IEEE, Vol. 2, pp. 1447-1454.

[6] Saif, J.A; Hammad, M.; Alqubati, I.A. (2016): Gradient Based Image Edge Detection. International Journal of Engineering and Technology, 8(3), pp.153.

[7] Sonka, M; Hlavac, V; Boyle R. (2008): Image Processing, Analysis and Machine Vision, Thomson.

[8] Xu, L; Lu, C ; Xu, Y; Jia, J. (2011): Image smoothing via L0 gradient minimization. In ACM Transactions on Graphics (TOG) ,30(6), pp. 174. 